\title{
MIGRATION OF TRANS-NEPTUNIAN OBJECTS TO THE EARTH
}

\author{
SERGEI I. IPATOV \\ Institute of Applied Mathematics, Miusskaya sq. 4, \\ Moscow 125047,Russia; E-mail: ipatov@spp.keldysh.ru
}

\begin{abstract}
Migration of trans-Neptunian objects under their mutual gravitation influence and the influence of the giant planets is investigated. These investigations are based on computer simulation results and on some formulas. We estimated that about $20 \%$ of near-Earth objects with diameter $d \geq 1 \mathrm{~km}$ may have come from the Edgeworth-Kuiper belt.
\end{abstract}

\section{Introduction}

The first object of the Edgeworth-Kuiper belt (EKB) was found in 1992, and now orbits of about 90 trans-Neptunian objects (TNOs) are known. The object 1996 $\mathrm{TL}_{66}$ with diameter $d \approx 500 \mathrm{~km}$ moves around the Sun at a larger distance than other TNOs. It's orbital elements are $a=85 \mathrm{AU}, e=0.6$, and $i=24^{\circ}$. Luu et al. (1997) considered that there are about $10^{4}$ such objects between 40 and $200 \mathrm{AU}$ with a total mass of $0.5 m_{\oplus}$, where $m_{\oplus}$ is the mass of the Earth. Semimajor axes of other observed TNOs lie between 35 and 49 AU. Their diameters are between 100 and $400 \mathrm{~km}$, and their characteristic mass is about $5 \cdot 10^{-12} M_{\odot}$, where $M_{\odot}$ is the mass of the Sun. Jewitt et al. (1996) and Levison and Duncan (1997) supposed that the inferred number of such objects is 70,000. Jewitt et al. (1996) estimated the total mass of the present-day belt in the 30-50 AU region to be about $(0.06-0.25) m_{\oplus}$ at $a \sim 30-50$ AU. As Pluto, approximately $35 \%$ of the known TNOs reside in the 2:3 resonance with Neptune, but Jewitt et al. (1998) concluded that this fraction is overrepresented as a result of observational selection and only $10-20$ $\%$ of TNOs in the 30-50 AU region are Plutinos. Jewitt et al. (1996) assumed that for the $100-400 \mathrm{~km}$ diameter objects the differential size distribution obey $n(r) d r=\Gamma r^{-q} d r$, with $\Gamma=$ const and $q=3$. The number of bodies with $d>1 \mathrm{~km}$ is predicted to be about $10^{10}$ for $30 \leq a \leq 50 \mathrm{AU}$. Weismann (1995) supposed that the dynamically inactive region beyond $45 \mathrm{AU}$ may extend out to $1000 \mathrm{AU}$ or more and may contain up to several times $10^{13}$ objects with the total mass of several hundred Earth masses.

The asteroid and Edgeworth-Kuiper belts are considered to be the main sources of near-Earth objects (NEOs). Collisions of some NEOs (such as the Tunguska object) with the Earth can destroy a large city. The comet origin of some NEOs and meteorites was suggested by Öpik (1963). According to Wetherill $(1988,1989)$ and Weissman et al. (1989), it is difficult to explain the number of objects of the Apollo and Amor groups and features of their orbits (for example, their mean inclinations, which are larger than those in the main asteroid belt), if one considers only asteroidal sources. Wetherill (1991) considered that NEOs should come from the EKB, but not from the Oort cloud in order to supply present inclinations of orbits of NEOs. Fernandez (1980) first supposed that the trans-Neptunian belt is a source of short-period comets. 
Many papers devoted to the EKB were published last years (see a review by Weissman (1995) and a later paper by Levison and Duncan (1997)). Stern (1995, 1996a,b), Davis and Farinella (1997) considered the collisional evolution of TNOs. It was obtained that the population of bodies with $d>100 \mathrm{~km}$ did not change much during the age of the Solar System. Most of bodies with diameter of several kilometers are debris of larger bodies. Davis and Farinella (1997) considered that collisions of TNOs can provide a significant influx of Chiron-sized bodies.

Migration of various bodies under the gravitational influence of planets was considered by Duncan et al. (1988), Hahn and Bailey (1990), Levison and Duncan (1994), and many other authors. Initial orbits of most of these bodies crossed the orbits of the giant planets. The median dynamical lifetime of short-period comets was obtained to be equal to $0.45 \mathrm{Myr}$ and that for Chiron-type objects is about 1.1-1.4 Myr.

The main aim of the present paper is to appreciate the number of TNOs migrating to the Earth.

\section{Interactions of Trans-Neptunian Objects}

Ipatov (1980, 1988, 1995a,b, 1997b, 1999a) considered variations in $a$ and $e$ of bodies due to the gravitational influence of the large $(d>100 \mathrm{~km})$ TNOs using new formulas for characteristic times elapsed up to close encounters. These formulas are different from those for the Öpik's approach used by other scientists. The case of variable inclinations was also considered. The evolution of three gravitating bodies with masses equal to that of Pluto was investigated by Ipatov (1980) with the use of a spheres' method and by Ipatov (1988) by numerical integration. The sphere of action (i.e., the Tisserand sphere) of radius $r_{s a} \approx R\left(m / M_{\odot}\right)^{2 / 5}$ was used, where $R$ is a distance from the Sun, $m$ is the mass of a TNO. Ipatov (1995a,b, 1997b, 1999a) made some appraisals of the evolution of a disk of TNOs. Basing on this appraisals, below we make some conclusions about interactions and the origin of TNOs.

Fernandez (1980), Ip and Fernandez (1997) in their investigations of mutual gravitational influence of TNOs used spheres smaller than $r_{s a}$ and so decreased the role of mutual gravitational influence. Fernandez (1980) investigated the variations in perihelion distance and made his calculations when masses of TNOs were unknown. Ip and Fernandez (1997) considered the gravitational influence of TNOs only on the orbital evolution of bodies located in the 2:3 resonance with Neptune.

We obtained that the characteristic time elapsed up to a close encounter (up to $r_{s a}$ ) for two objects with masses $m \sim 5 \cdot 10^{-12} M_{\odot}$ is equal to $7 \cdot 10^{10} \mathrm{yr}$. If there are about $7 \cdot 10^{4}$ objects with diameter $d \geq 100 \mathrm{~km}$ in the belt, then during the age $T_{s s}$ of the Solar System a body with a semimajor axis $a \approx 40$ AU takes part in about 3000 close encounters with TNOs with $d \geq 100 \mathrm{~km}$. The probability of that during $T_{s s}$ an object with $d \geq 100 \mathrm{~km}$ collided with one of objects of the same size equals to 0.005 . If the number of objects with $d \geq 10 \mathrm{~km}$ is greater than that 
with $d \geq 100 \mathrm{~km}$ by a factor of 100 , then a probability of a collision during $T_{s s}$ of an object with $d \geq 100 \mathrm{~km}$ with some object with $d \geq 10 \mathrm{~km}$ equals to 0.15 . As the average eccentricity of TNOs is about 0.1 , variations in $a$ for smaller colliding bodies can exceed several AU. Probably, less than $1 \%$ of $100-\mathrm{km}$ bodies had such large variations in $a$ due to collisions during $T_{s s}$.

Our numerical estimates showed that the mean variation in $a$ at one close encounter is $\delta a \sim(1-3) \cdot 10^{-5} a$ for $m=5 \cdot 10^{-12} M_{\odot}$ and mean eccentricities and inclinations of the observed TNOs. The variation in $a$ of an object located in the middle of the EKB probably is proportional to $\delta a \sqrt{N}$ (where $N$ is the number of close encounters of this object with large TNOs) and usually does not exceed $0.1 \mathrm{AU}$ during $T_{s s}$. For many objects at the inner part of the belt (at $a<39 \mathrm{AU}$ ), $a$ could vary more monotonously and could decrease by more than 1 AU during $T_{s s}$. Such decrease of $a$ may be a reason of that a region ( $\left.36 \leq a \leq 39 \mathrm{AU}\right)$ with small values of $e$ and $i$ is unpopulated, though, as it was shown by Duncan et al. (1995), it is dynamically stable under the gravitational influence of planets for $T_{s s}$. At some very close encounters, semimajor axes of TNOs could change by several AU. Several percents of TNOs could take part in such very close encounters during $T_{s s}$. The number of such close encounters is, by an order of magnitude, larger than the corresponding number of collisions, and so the role of gravitational interactions in variations of orbital elements is larger than that of collisions. On average, during $T_{s s}$ a TNO had several close (up to $r_{s}$ ) encounters with Pluto and changed its $a$ by $\sim 0.1 \mathrm{AU}$ at these encounters.

Due to the mutual gravitational influence of TNOs, their mean eccentricity and mean inclination could not reach their present values at the present-day mass of the EKB, but could reach them only for a total mass of the belt of about several $m_{\oplus}$ (Ipatov, 1995a,b). The results of numerical simulation of the process of planetary accretion (Ipatov, 1987, 1993) showed that the total mass of planetesimals that entered the trans-Neptunian region from the feeding zone of the giant planets during their accumulation could exceed tens $m_{\oplus}$. These planetesimals could increase initially small eccentricities of 'local' TNOs formed in the EKB, and swept most of the local TNOs, which total initial mass $M_{\Sigma}$ could exceed $10 m_{\oplus}$. A small part of such planetesimals could left beyond the orbit of Neptune in eccentric orbits (such as that of $1996 \mathrm{TL}_{66}$ ).

Eneev (1980) supposed that large TNOs were formed from rarefied ice-gas condensations and smaller objects are their debris. Stern (1996a), Stern and Colwell (1997) simulated the formation of $100-1000 \mathrm{~km}$ objects from the 1-10 km planetesimals. They obtained that such objects could be formed only for $M_{\Sigma}>10 m_{\oplus}$ and $e<0.002$. Kenyon and Luu (1998) obtained runaway growth in $100 \mathrm{Myr}$ for $e=0.001$ and in 700-2000 Myr for $e=0.01$ at $M_{\Sigma} \geq 10 m_{\oplus}$. These times are greater than the time of formation of the massive Jupiter, which does not exceed several tens of Myr. We consider that such small eccentricities could not exist during a large time span, both due to the mutual gravitational influence of TNOs and due to the gravitational influence of the forming giant planets. Therefore, probably, 
most of large $(d \geq 100 \mathrm{~km})$ local TNOs were formed mainly by the compression of condensations but not by the accumulation of smaller planetesimals. Some smaller objects could be formed directly from condensations and a considerable part of them are debris of larger objects.

\section{Migration of Trans-Neptunian Objects under the Gravitational Influence of the Giant Planets}

Duncan et al. (1995) found regions of the values of $a$ and $e$, for which TNOs can migrate to the orbit of Neptune under the gravitational influence of planets. Gladman and Duncan (1990), Torbett and Smoluchowski (1990) found that due to gravitational influence of the giant planets the orbits of bodies of the inner part of the EKB could begin to cross the orbit of Neptune. Holman and Wisdom (1993), Levison and Duncan (1993), and Duncan et al. (1995) investigated times survived by test TNOs before they became Neptune-crossers. They finished their calculations of the evolution of test TNOs when their orbits began to cross the orbit of Neptune or the body entered inside the Hill sphere of Neptune. Levison and Duncan (1997) considered the motion of TNOs to the Jupiter's orbit. Morbidelli et al. (1995) investigated evolution of some resonant TNOs that did not get deep inside the Solar System.

Ipatov (1997a) considered migration of objects from the EKB under the gravitational influence of the giant planets not only to the orbits of Neptune and Jupiter, as other authors, but also further to the orbit of the Earth. Below we present more wide results of these runs. The gravitational influence of the giant planets was taken into account with the use of the RMVS2 program of symplectic method from the Swift integration package worked out by Levison and Duncan (1994). The time step was the same as in their test, i.e., equaled to $1 \mathrm{yr}$. Initial orbits of planets also were taken from the test. This integrator is by an order of magnitude faster than previous methods of integration. We considered various initial eccentricities $e_{0}$, inclinations $i_{\circ}$, and orbital orientations of orbits. Initial values $a_{0}$ of semimajor axis were varied from 35 to $50 \mathrm{AU}$. Orbital evolution of one hundred bodies was considered. The considered time span $T$ usually equaled to $20 \mathrm{Myr}$. In some runs $T$ reached $100-150 \mathrm{Myr}$.

In the case without close encounters for some typical orbits, we compared results obtained with the use of the RMVS2 integrator with those obtained with the integrator by Bulirsh and Stoer (1966). The plots of variations in orbital elements with time are presented by Ipatov (1999b). It was shown that limits of variations in $a$ during 1 Myr differed by less than $5 \%$, and differences in $e$ and $i$ were smaller. A more detailed comparison of the results obtained by these integrators is presented below in section 4 .

We found that some bodies of the EKB can migrate deep inside the Solar System. The minimal perihelion distance of 2, 8, and 10 considered migrating bodies was in the regions $[6,9.5],(9.5,20)$, and $[20,30) \mathrm{AU}$, respectively. Four 


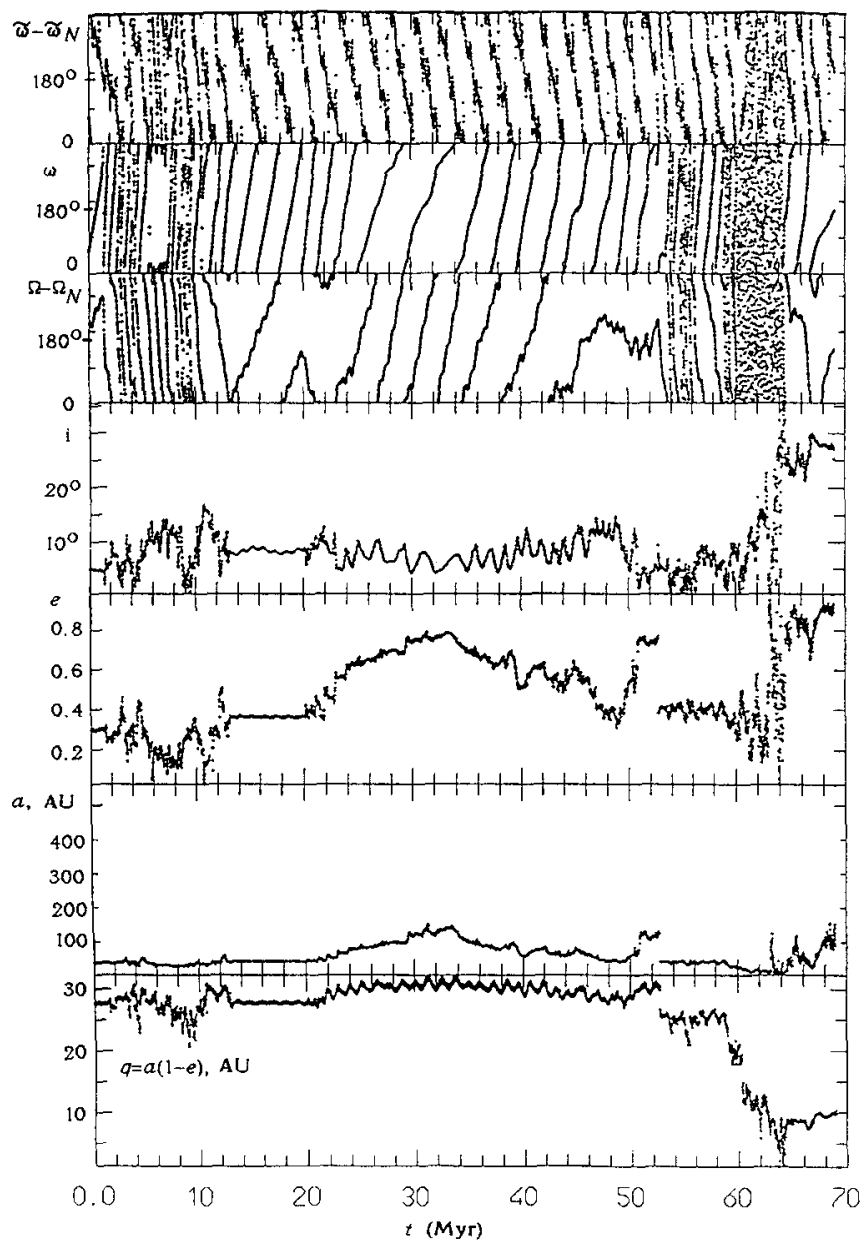

Fig. 1. Time variations in the semimajor axis $a$, eccentricity $e$, perihelion distance $q=a(1-e)$, inclination $i$, the difference between the longitudes of the ascending node for a body and Neptune, $\Delta \Omega=\Omega-\Omega_{N}$, the argument of perihelia, $\omega$, and the difference between the longitudes of perihelia for a body and Neptune, $\Delta \tilde{\omega}=\tilde{\omega}-\tilde{\omega}_{N}$. Initial data: $a_{\circ}=40 \mathrm{AU}, e_{0}=0.15, i_{0}=5^{\circ}, \Omega_{0}=\omega_{0}=M_{0}=60^{\circ}$.

bodies reached the Jupiter's orbit during evolution and two of them almost reached the Earth's orbit. At $i_{\circ}=5^{\circ}$ and initial values of the longitude of ascending node, the argument of perihelion, and the mean anomaly equal to $\Omega_{\circ}=\omega_{0}=M_{\circ}=60^{\circ}$, for $a_{\circ}=40 \mathrm{AU}$ and $e_{0}=0.15$ and for $a_{\circ}=39.3 \mathrm{AU}$ and $e_{0}=0.3$, the perihelion distance $q$ decreased from 34 and 27.5 AU to 1.25 and $1.34 \mathrm{AU}$ in 25 and $64 \mathrm{Myr}$, and these bodies were ejected into hyperbolic orbits in 30 and $70 \mathrm{Myr}$, respectively (Figs. 1 and 2). Maximum inclinations $i_{\max }$ in these runs were equaled to $57^{\circ}$ and 


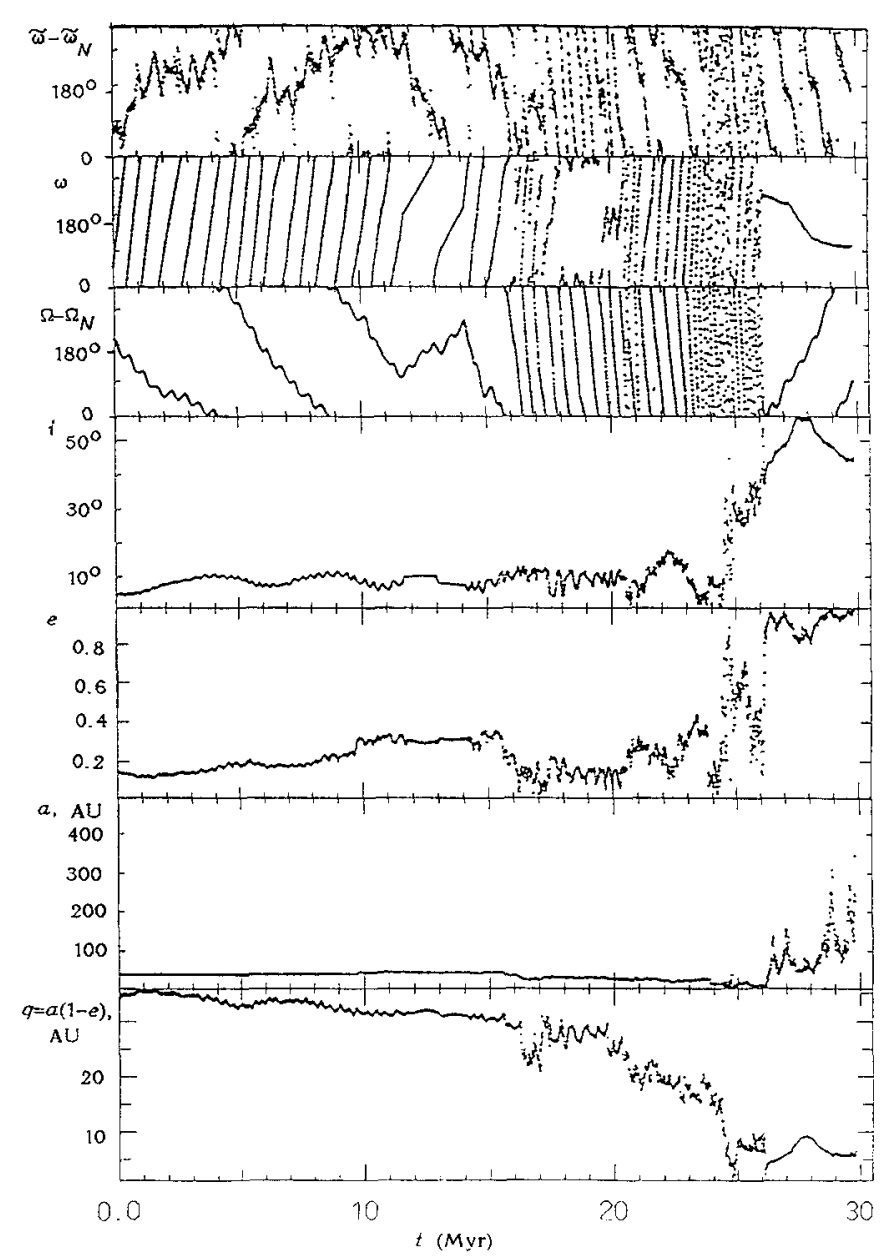

Fig. 2. Same as figure 1; initial data: $a_{0}=39.3 \mathrm{AU}, e_{0}=0.3, i_{0}=5^{\circ}$, $\Omega_{0}=\omega_{0}=M_{0}=60^{\circ}$.

$36^{\circ}$, respectively, and there were large variations in $e$ and $i$ when $q$ was close to minimum. As orbital elements were sampled with a time step $\Delta t=20,000 \mathrm{yr}$, the actual minimal values of $q$ can be smaller than those presented above. The time interval, during which $q$ decreased from 10 to $1.3 \mathrm{AU}$, equaled to $0.3-0.5 \mathrm{Myr}$, and that for $q$ decreased from $5 \mathrm{AU}$ to $1.3 \mathrm{AU}$ was considerably smaller. Plots for some other initial data are presented by Ipatov (1999b). For one run at $a_{0}=39.3 \mathrm{AU}$, $e_{\circ}=0.15$, and $i_{\circ}=5^{\circ}$, the time interval, during which $q$ decreased from $30 \mathrm{AU}$ to $3 \mathrm{AU}$, was less than $2 \mathrm{Myr}$.

For some runs $q$ exceeded $10 \mathrm{AU}$ during all time span before the ejection of 
a body into a hyperbolic orbit. For example, at $a_{\mathrm{o}}=39.3 \mathrm{AU}, e_{\mathrm{o}}=0.15$, and $i_{\mathrm{o}}=5^{\circ}$, a test body was ejected into a hyperbolic orbit after $41.5 \mathrm{Myr}$, and $q>15$ $\mathrm{AU}$ and $i \leq 33^{\circ}$ during this time span. For $a_{\mathrm{o}}=40 \mathrm{AU}, e_{\mathrm{o}}=0.05$, and $i_{\mathrm{o}}=5^{\circ}$, a test body was in an elliptical orbit with $q>10 \mathrm{AU}$ during the considered time span $T=100 \mathrm{Myr}$, though the variations in orbital elements were large and maximum eccentricity $e_{\max }$ exceeded 0.8. For $a_{\mathrm{o}}=38.9 \mathrm{AU}, e_{\mathrm{o}}=0.15, i_{\mathrm{o}}=5^{\circ}$, and $T=150 \mathrm{Myr}$, we have $q \geq 15 \mathrm{AU}, e_{\max }=0.76$, and $i_{\max }=27^{\circ}$. During the first $30 \mathrm{Myr}$, variations in $a$ were small in this run. For larger values of $e_{0}$, the portion of bodies that cross the orbit of Neptune during evolution is larger. The time $T_{h}$ elapsed until the ejection of a body into a hyperbolic orbit is smaller, as a rule, for smaller values of $i_{\mathrm{o}}$. For example, at $a_{\mathrm{o}}=39.3 \mathrm{AU}$ and $e_{\mathrm{o}}=0.3$, it was obtained that $T_{h} \approx 70 \mathrm{Myr}$ for $i_{\circ}=5^{\circ}$ and $T_{h} \approx 7 \mathrm{Myr}$ for $i_{\circ}=0$.

Limits of variations in $a, e$, and $i$ may differ considerably for runs with the same values of $a_{0}, e_{\circ}$, and $i_{\circ}$, but with different values of $\Omega_{0}, \omega_{\circ}$, and $M_{0}$. Such influence of initial orbital orientations was obtained both for resonant and nonresonant values of $a_{\circ}$. For example, at $a_{\circ}=39.3 \mathrm{AU}, e_{\circ}=0.15$, and $i_{\circ}=5^{\circ}$, we investigated evolution of 12 orbits with various values of $\Omega_{\mathrm{o}}, \omega_{\mathrm{o}}$, and $M_{\circ}$ during $T=20 \mathrm{Myr}$. All these objects moved in the 2:3 resonance with Neptune. It was obtained that $e_{\max }<0.2$ for 5 runs and $e_{\max }>0.3$ for 3 runs. For nonresonant value $a_{\circ}=40$ AU at $e_{0}=0.15, i_{\circ}=5^{\circ}, \omega_{0}=M_{\circ}=60^{\circ}$ and the same time span, we obtained $e_{\max } \approx e_{\mathrm{o}}=0.15$ at $\Omega_{\mathrm{o}}=0$ and $e_{\max }=0.36$ at $\Omega_{\mathrm{o}}=60^{\circ}$. For $\Omega_{\mathrm{o}}=60^{\circ}$, the object was in the $\nu_{8}$ resonance at $10 \leq t \leq 12 \mathrm{Myr}$ and in the Kozai resonance at $18 \leq t \leq 20 \mathrm{Myr}$. For $a_{\circ}=42 \mathrm{AU}, e_{\mathrm{o}}=0.05, i_{\circ}=5^{\circ}$ and $T=20 \mathrm{Myr}$, we have $e_{\max }=0.07, i_{\max }=5.7^{\circ}$, and the minimum value of $q$ is equal to $q_{\min }=39$ $\mathrm{AU}$ at $\Omega_{\circ}=\omega_{\circ}=M_{\circ}=60^{\circ}$, and $e_{\max }=0.33, i_{\max }=9.6^{\circ}$, and $q_{\min }=31 \mathrm{AU}$ at $\Omega_{\mathrm{o}}=\omega_{\mathrm{o}}=M_{\mathrm{o}}=0$. For the last run at $t<10 \mathrm{Myr}$, the object was in the $\nu_{18}$ resonance $\left(\Omega-\Omega_{N}\right.$ librated around $180^{\circ}$, where $\Omega_{N}$ is the value for Neptune), $\tilde{\omega}-\tilde{\omega}_{N}(\tilde{\omega}=\Omega+\omega)$ varied between $120^{\circ}$ and $370^{\circ}$, and $e$ grew monotonously from 0.05 to 0.2 . The above examples show that limits of variations in orbital elements of TNOs can highly depend on their initial orientations of orbits. Therefore, small variations in orbital elements due to collisions and mutual gravitational influence of TNOs can cause large variations in orbits under the gravitational influence of the giant planets.

For all considered runs and $T=20 \mathrm{Myr}$, the variations in $a$ and maximum values of $e$ and $i$ during evolution exceeded $0.6 \mathrm{AU}, 0.05$ and $3^{\circ}$, respectively. Therefore, the values of $e$ and $i$ of TNOs can not be small for a long time.

Several types of variations in orbital elements for the $2: 3$ resonance with Neptune were considered by Ipatov and Henrard (1999). Ipatov $(1995 b, 1996)$ investigated migration of small bodies under the gravitational influence of all planets with the use of the method of spheres of action. Hundreds of bodies were considered in these runs, and it was obtained that individual bodies decreased their aphelion distances $Q$ from the trans-Neptunian zone to the values even less than $1 \mathrm{AU}$. The first object (1998 $\mathrm{DK}_{36}$ ) with $Q \leq 1 \mathrm{AU}$ was found in 1998. For $1998 \mathrm{XX}_{2}$ and 
$1999 \mathrm{AO}_{10}, Q \approx 1.013 \mathrm{AU}$.

\section{The Number of Trans-Neptunian Objects Reaching the Orbit of the Earth}

The estimates of the number of TNOs migrating to the Earth can be made on the basis of simple formulas and results of numerical integration. The number of TNOs, which reached the Jupiter's orbit during the considered time span $T$, equals to $N_{J}=N \cdot P_{N} \cdot p_{J N}$, where $N$ is the number of objects in the belt, $P_{N}$ is the portion of TNOs migrating to the Neptune's orbit and leaving the belt during this time $T$, and $p_{J N}$ is the portion of Neptune-crossing objects leaving the belt, which reaches the Jupiter's orbit during their lifetimes. The number of TNOs, which are now Jupiter-crossers, equals to $N_{J_{n}}=N_{J} \Delta t_{J} / T$, where $\Delta t_{J}$ is the average time, during which the object crosses the Jupiter's orbit. Our results of numerical simulations showed that $\Delta t_{J} \approx 0.2 \mathrm{Myr}$.

It is considered that there are about $10^{10}$ objects with $d>1 \mathrm{~km}$ and $30 \leq a \leq 50$ AU. Duncan et al. (1995) obtained that the portion $P_{N}$ of TNOs that left the EKB during $4 \cdot 10^{9}$ yr under the influence of the giant planets is about $0.1-0.2$. As mutual gravitational influence of TNOs also takes place, we take this portion equal to 0.2. The portion of Neptune-crossing objects, which reach the Jupiter's orbit during their lifetimes, was obtained by Duncan et al. (1995) to be equal to 0.34. So we have $N_{J}=6.8 \cdot 10^{8}$ for $T=4 \cdot 10^{9} \mathrm{yr}$ and $N=10^{10}$, and also $N_{J_{n}}=3.4 \cdot 10^{4}$ at $\Delta t_{J}=0.2 \mathrm{Myr}$.

The number of TNOs, which reached the orbit of the Earth during the considered time $T$, equals to $N_{E}=N_{J} \cdot p_{J E}=N \cdot P_{N} \cdot p_{J N} \cdot p_{J E}$, where $p_{J E}$ is the portion of Jupiter-crossing objects that reach the orbit of the Earth during their lifetimes. The ratio of the number $N_{E N}$ of Earth-crossing objects (ECOs), which came from the EKB, to the total number $N_{E C O}$ of ECOs at the considered time moment equals to $P_{N E}=N_{E N} / N_{E C O}=N \cdot P_{N} \cdot p_{J N} \cdot p_{J E} \cdot \Delta t_{E} /\left(T \cdot N_{E C O}\right)$, where $N_{N E}=N_{E} \cdot \Delta t_{E} / T, \Delta t_{E}$ is the mean time, during which a Jupiter-crossing object crosses the orbit of the Earth. The number of collisions of TNOs with the Earth during time $T$ equals to $N_{\text {col }}=N_{E} \cdot \Delta t_{E} / T_{E}$, where $T_{E}$ is a characteristic time elapsed up to a collision of an ECO with the Earth.

Ipatov and Hahn (1997, 1999a, 1999b) investigated the evolution of orbits close to the orbit of the object P/1996 R2 (Lagerkvist) under the gravitational influence of all planets. This object has a Jupiter-crossing orbit $(a \approx 3.79 \mathrm{AU}, e \approx 0.31$, $i \approx 2.6^{\circ}$ ). Results of investigations with the use of the RMVS3 integrator (which is the modification of the RMVS2 integrator) from the Swift package by Levison and Duncan (1994) for initial integration step equal to 30 days were close on the whole to those obtained with the BULSTO integrator by Bulirsh and Stoer (1966). Median lifetimes (before ejections into hyperbolic orbits) in series of 12 runs were equal to several hundreds of thousands years. Using the BULSTO integrator, we obtained that the minimal distance from the Sun $q_{\min }<1$ AU for 8 among 24 runs (i.e., for $33 \%$ of runs) for integration into the future and for 2 among 24 runs for 
integration into the past (in total, this inequality is fulfiled for 10 among 48 runs, i.e., for about $20 \%$ ). Using the RMVS3 integrator, we obtained $q_{\min }<1$ AU for 3 among 24 runs and for 1 among 24 runs for integration into the future and into the past, respectively. In our runs Jupiter-crossing bodies reached the orbit of the Earth more rarely for the RMVS3 integrator. It is mainly caused by the main difference between the results obtained by the RMVS3 and BULSTO integrators, which is that test bodies more often moved in resonances with planets, when we used the BULSTO integrator. So actual migration of TNOs to the Earth can be even larger than that obtained with the use of the RMVS2 integrator and presented in Section 3.

Basing on the above runs, we estimated $p_{J E}=0.2$ and $\Delta t_{E} \approx 5000 \mathrm{yr}$. According to (Ipatov, $1995 \mathrm{~b}$ ), $T_{E} \approx 75 \mathrm{Myr}$, and so the probability of a collision of an ECO with the Earth during $5000 \mathrm{yr}$ was obtained to be about $7 \cdot 10^{-5}$. It is considered that there are about 500-1000 ECOs with $d>1 \mathrm{~km}$. For $d \geq 1$ $\mathrm{km}, N_{E C O}=750$, and $T=4 \cdot 10^{9} \mathrm{yr}$, we have $P_{N E} \approx 0.2, N_{E} \approx 1.4 \cdot 10^{8}$, $N_{N E} \approx 170$, and $N_{\text {col }} \approx 10^{4}$. For $d \geq 100 \mathrm{~km}$ and $N=7 \cdot 10^{4}$, the probability of a collision with the Earth during $T=4 \cdot 10^{9} \mathrm{yr}$ is about 0.05 . The above estimates are very approximate, but they show that the number of TNOs hitting the Earth is not small.

As it is more easy to destruct icy bodies than stone or metal bodies (for example, bodies can break up at close encounters with planets on their way to the Earth), the portion of TNOs among ECOs for bodies with $d \leq 100 \mathrm{~m}$ (for example, for Tunguska-type bodies) may be greater than that for $1 \mathrm{~km}$ bodies and can exceed 20 $\%$, but small icy bodies disappear in the atmosphere and can not reach the surface of the Earth. The above estimates were obtained for bodies, which migrated from distances 30-50 AU from the Sun. Bodies with $a>50 \mathrm{AU}$ moving in highly eccentric orbits also can migrate to the Earth.

\section{Conclusion}

Probably, TNOs with $d>100 \mathrm{~km}$ moving in low eccentrical orbits were formed directly by the compression of large rarefied condensations, but not by the accretion of smaller solid planetesimals. Small variations in orbital elements of TNOs due to their mutual gravitational influence can cause large orbital variations due to gravitational influence of the giant planets. About $20 \%$ of NEOs with $d \geq 1 \mathrm{~km}$ may have come from the EKB.

\section{Acknowledgements}

This work was supported by the Russian Foundation for Basic Research, project no 96-02-17892, and by the Russian federal scientific and technical program Astronomy. Computer simulations of the orbital evolution of trans-Neptunian objects under the gravitational influence of the giant planets were made in 1995 during the visit to FUNDP (Namur, Belgium) supported by the ESO grant no. B-06-018. 


\section{References}

Bulirsh, R. and Stoer, J.: 1966, Numer. Math., 8, 1-13.

Davis, D.R. and Farinella, P.: 1997, Icarus, 125, 50-60.

Duncan, M., Quinn, T., and Tremaine, S.: 1988, 328, L69-L73.

Duncan, M.J., Levison, H.F., and Budd, S.M.: 1995, Astron. J., 110, 3073-3081.

Eneev, T.M.: 1980, Sov. Astron. Letters, 6, No 5, p. 295-300 in Russian edition.

Fernandez, J.A.:1980, Monthly Notices Roy. Astron. Soc., 192, 481-491.

Gladman, B. and Duncan, M.: 1990, Astron. J., 100, 1680-1696.

Hahn, G. and Bailey, M.E.: 1990, Nature, 348, 132-136.

Holman, M.J. and Wisdom, J.: 1993, Astron. J., 105, 1987-1999.

Ip, W.-H. and Fernandez, J.A.: 1997, Astron. Astrophys., 324, 778-784.

Ipatov, S.I.: 1980, Preprint of Inst. of Applied Mathematics, N 43, Moscow, 33 P. (in Russian).

Ipatov, S.I: 1987, Earth, Moon, and Planets, 39, 101-128.

Ipatov, S.I.: 1988, Kinematics Phys. Celest. Bodies, 4, N 6, 76-82.

Ipatov, S.I.: 1993, Solar System Research, 27, 65-79.

Ipatov, S.I.: 1995a, Solar System Research, 29, 9-20.

Ipatov, S.I.: 1995b, Solar System Research, 29, 261-286.

Ipatov, S.I.: 1996, Earth, Moon, and Planets, 72, 211-214.

Ipatov, S.I.: 1997a, LPSC XXVIII, 615-616.

Ipatov, S.I.: 1997b, LPSC XXVIII, 617-618.

Ipatov, S.I.: 1999a, 'Migration of Kuiper-belt objects inside the Solar System', "Planetary systems

- the long view", Proc. 9th Rencontres de Blois (June 22-28, 1997), Editions Frontieres, Gif.

sur Yvette Cedex, Celnikier, L.M. and Tran Thanh Van, J., (eds.), 157-160

Ipatov, S.I.: 1999b, Migration of celestial bodies in the solar system, URSS Publishing Company, Moscow, in press.

Ipatov, S.I. and Hahn, G.J.: 1997, LPSC XXVIII, 619-620.

Ipatov, S.I. and Hahn, G.J.: 1999a, "Planetary systems - the long view", Proc. 9th Rencontres de Blois (June 22-28, 1997), Editions Frontieres, Gif. sur Yvette Cedex, Celnikier, L.M. and Tran Thanh Van, J., (eds.), 179-180

Ipatov, S.I. and Hahn, G.J.: 1999b, Solar System Research, 33, in press.

Ipatov, S.I. and Henrard, J.: 1999, Solar System Research, 33, in press.

Jewitt, D., Luu., J., and Chen J.: 1996, Astron. J., 112, 1225-1238.

Jewitt, D., Luu., J., and Trujillo, C.: 1998, Astron. J., 115, 2125-2135.

Kenyon, S.J. and Luu, J.X.: 1998, Astron. J., 115, 2136-2160.

Levison, H.F. and Duncan, M.J.: 1993, Astrophys. J., 406, L35-L38.

Levison, H.F. and Duncan, M.J.: 1994, Icarus, 108, 18-36.

Levison, H.F. and Duncan, M.J.: 1997, Icarus, 127, 13-23.

Luu, J., Marsden, B.G., Jewitt, D., Trujillo, C.A., Hergenrother, C.W., Chen, J., and Offutt, W.B.: 1997, Nature, 387, 573-575.

Morbidelli, A., Thomas, F., and Moons, M.: 1995, Icarus, 118, 322-340.

Öpik, E.J.: 1963, Adv. Astron. Astrophys., 2, 219-262.

Stern, S.A.: 1995, Astron. J., 110, 856-868.

Stern, S.A.: 1996a, Astron. J., 112, 1203-1211.

Stern, S.A.: 1996b, Astron. Astrophys., 310, 999-1010.

Stern, S.A. and Colwell, J.E.: 1997, Astron. J., 114, 841-849.

Torbett, M. and Smoluchowski, R.: 1990, Nature, 44, 722-729.

Weissman, P.R.: 1995, Annu. Rev. Astron. Astrophys., 33, 327-357.

Weissman, P.R., A'Hearn, M.F., McFadden, L.A. and Rickman, H.: 1989, Asteroids II, Tucson: Univ. Arizona Press, Binzel, R.P., Gehrels, T. and Matthews, M.S. (eds.), 880-919.

Wetherill, G.W.: 1988, Icarus, 76, 1-18.

Wetherill, G.W.: 1989, Meteoritics, 24, 15-22.

Wetherill, G.W.: 1991, Proc. of the IAU Colloquium 121 "Comets in the post-Halley era", Amsterdam: Kluwer Acad. Publ., Newburn, R.L., Rahe, J. and Neugebauer, M. (eds.), 537-556. 